\title{
Cognitive and White Matter Tract Differences in MS and Diffuse Neuropsychiatric Systemic Lupus Erythematosus
}

B. Cesar, M.G. Dwyer, J.L. Shucard, P. Polak, DN. Bergsland, R.H.B. Benedict, B. Weinstock-Guttman, D.W. Shucard, and R. Zivadinov

\begin{abstract}
BACKGROUND AND PURPOSE: Multiple sclerosis and neuropsychiatric systemic lupus erythematosus are autoimmune diseases with similar CNS inflammatory and neurodegenerative characteristics. Our aim was to investigate white matter tract changes and their association with cognitive function in patients with MS and those with neuropsychiatric systemic lupus erythematosus compared with healthy controls by using diffusion tensor imaging.
\end{abstract}

MATERIALS AND METHODS: Thirty patients with relapsing-remitting MS and 23 patients with neuropsychiatric systemic lupus erythematosus matched for disease severity and duration and 43 healthy controls were scanned with 3T MR imaging. The DTI was postprocessed, corrected for lesions, and analyzed with tract-based spatial statistics. Cognitive assessment included examination of processing speed; visual, auditory/verbal, and visual-spatial memory; and sustained attention and executive function. Differences were considered significant at $P<.05$.

RESULTS: Tract-based spatial statistics analysis revealed significantly decreased fractional anisotropy and increased mean diffusivity in patients with MS compared with healthy controls, decreased fractional anisotropy in patients with MS compared with those with neuropsychiatric systemic lupus erythematosus, and an increased mean diffusivity in patients with neuropsychiatric systemic lupus erythematosus compared with healthy controls. Patients with MS showed decreased fractional anisotropy throughout central WM pathways, including the corpus callosum and the inferior longitudinal and fronto-occipital fasciculi compared with those with neuropsychiatric systemic lupus erythematosus. Altered cognitive scores in patients with MS were significantly associated with decreased fractional anisotropy and increased mean diffusivity in all examined domains, while in patients with diffuse neuropsychiatric systemic lupus erythematosus, only decreased fractional anisotropy in the superior WM pathways showed significant association with executive function.

CONCLUSIONS: Patients with MS and neuropsychiatric systemic lupus erythematosus showed widespread WM tract alterations outside overt lesions, though more severe changes were identified in patients with MS. The WM tract changes were associated with cognitive dysfunction in all explored domains only in patients with MS.

ABBREVIATIONS: $A D=$ axial diffusivity; $F A=$ fractional anisotropy; $H C=$ healthy control; $M D=$ mean diffusivity; NPSLE = neuropsychiatric systemic lupus erythematosus; PASAT $=$ Paced Auditory Serial Addition Task; RD = radial diffusivity; SLE = systemic lupus erythematosus; TBSS $=$ tract-based spatial statistics

M ultiple sclerosis and systemic lupus erythematosus (SLE) are both chronic inflammatory autoimmune diseases that adversely affect the CNS. ${ }^{1}$ MS is characterized by both focal and diffuse damage in the brain and spinal cord due to demyelination, leading to axonopathy and tissue atrophy. While the pathology of MS is pri-

Received December 24, 2014; accepted after revision February 13, 2015.

From the Buffalo Neuroimaging Analysis Center, Department of Neurology (B.C., M.G.D., P.P., N.B., R.Z.), Department of Neurology (M.G.D., J.L.S., R.H.B.B., B.W.-G., D.W.S.), Division of Cognitive and Behavioral Neurosciences (B.C., J.L.S., D.W.S.), Neuroscience Program (J.L.S., D.W.S.), and MRI Clinical Translational Research Center (R.Z.), School of Medicine and Biomedical Sciences, University at Buffalo, State University of New York, Buffalo, New York; and Magnetic Resonance Laboratory (N.B.), IRCCS Don Gnocchi Foundation, Milan, Italy.

This work was supported in part by National Institutes of Health Grant NS049111 to J.L.S. marily restricted to the CNS, SLE can involve multiple organ systems. CNS involvement in SLE is mainly attributed to autoantibody attack on small vessels, resulting in diffuse ischemic damage. However, axonal loss and demyelination may also result from direct antibody attack on neuronal cells, either on neuronal bodies or in the WM tracts. $^{2}$

Although SLE and MS have notable differences in etiology, there is a marked overlap concerning the CNS inflammatory and neurodegenerative nature of these 2 diseases. $^{3}$ In 1972, Fulford et

Please address correspondence to Robert Zivadinov, MD, PhD, FAAN, Department of Neurology, School of Medicine and Biomedical Sciences, 100 High St, Buffalo, NY 14203; e-mail: rzivadinov@bnac.net

- Indicates open access to non-subscribers at www.ajnr.org

http://dx.doi.org/10.3174/ajnr.A4354 
$\mathrm{al}^{4}$ first coined the term "lupoid sclerosis" when referring to patients with SLE who presented with symptoms similar to those in MS. Additionally, neuropsychological and cognitive testing revealed similarities in cognitive profiles of patients with MS and those with SLE that may be the result of similar dysfunctional CNS structures. ${ }^{5,6}$ These neuropsychological findings included deficits in working memory and processing speed and general lower cognitive scores, even in the absence of a diagnosed neurologic or psychiatric condition. ${ }^{7,8}$ It is possible that these cognitive disturbances may be attributed to decreased WM integrity, the presence and extent of WM lesions, or GM damage, all of which are present in patients with MS and those with SLE. ${ }^{2,3,8-10}$

Unlike in MS, the relationship between cerebral pathology and resulting neuropsychiatric disorders in SLE is not well-established. Neuropsychiatric symptoms range from transient to chronic and are heterogeneous among patients. ${ }^{3,6}$ The American College of Rheumatology has identified 19 different syndromes of neuropsychiatric systemic lupus erythematosus (NPSLE). NPSLE includes focal syndromes such as seizures, strokes, and transient ischemic attacks and also diffuse syndromes such as anxiety disorders, mood disorders, headache, and cognitive impairment. ${ }^{11}$ Since the establishment of a separate NPSLE diagnosis, more studies have concentrated on using the NPSLE population for further inquiry into neurocognitive dysfunction. In a 1-year longitudinal study, subjects with NPSLE were found to have cognitive impairment associated with GM and WM dysfunction. ${ }^{12} \mathrm{Sev}-$ eral other studies have associated cognitive impairment with WM abnormalities in patients with NPSLE. ${ }^{13}$

The present study investigated the integrity of WM tracts adjusted for lesions by comparing DTI anisotropy in patients with NPSLE with diffuse syndromes, excluding patients with the defined focal syndromes, versus a group of patients with MS and a healthy control (HC) group. We used the voxel-based DTI tractbased spatial statistics (TBSS) (fMRI of the Brain Software Library [FSL]; http://fsl.fmrib.ox.ac.uk/fsl/fslwiki/TBSS $)^{14}$ method to reveal subtle changes along WM tracts. We also derived global WM measures to show an averaged measure of diffusivity in the brain. Because the MS disease process involves predominantly demyelination of the WM tracts, we hypothesized that patients with MS would have significantly lower DTI fractional anisotropy (FA) values and increased mean diffusivity (MD) values (measures of WM tract integrity) compared with patients with NPSLE and that patients with MS and those with NPSLE would have significantly lower FA and higher MD compared with HCs. We also explored the association between WM tract changes and cognitive function within study groups. We hypothesized that decreased WM tract integrity, indicated by increased MD and decreased FA, would be associated with poorer cognitive function.

\section{MATERIALS AND METHODS Subjects}

Participants were 30 patients with relapsing-remitting MS, 23 patients with NPSLE with diffuse syndromes, and 43 HCs. The outpatient groups were matched for disease duration (MS, mean of 11.7 years; NPSLE, mean of 15 years), and all patients had mildto-moderate disease severity. Patients with NPSLE and those with MS were recruited from the specialty centers in a local hospital and rheumatologists in the area. The diagnosis of SLE was independently confirmed by a rheumatology specialist and clinical routine laboratory testing obtained from medical records, whereas the MS diagnosis was confirmed by a neurology specialist. HC participants were recruited from advertisements in a local newspaper. All participants were screened for general selection criteria. The participants were then tested further for other exclusionary criteria such as a history of head trauma, learning disability, alcohol and drug use, notable hearing loss, visual problems, or other medical conditions unrelated to MS and SLE that could affect cognition. HC participants were excluded if they had a $\mathrm{Di}$ agnostic and Statistical Manual of Mental Disorders, 4th ed Axis I psychiatric disorder or if they were on psychotropic or any other medications that could affect cognition.

All subjects met the criteria for MR imaging testing based on a health-screening interview and an MR imaging questionnaire containing information about medical history (medications, surgeries, pregnancy, illness, and so forth). The Expanded Disability Status Scale was used to measure disease severity in patients with MS and was obtained at a 30-day interval from cognitive testing. Disease activity at the time of testing was measured for patients with NPSLE with a checklist based on the Systemic Lupus Activity Measure, ${ }^{15}$ with scores ranging from 0 to 19 , and was calculated as the sum of positive responses on a checklist of current symptoms (including fever, hair loss, joint pain, and so forth). The determination of cognitive impairment was based on the American College of Rheumatology guidelines established by the cognition subcommittee of the Ad Hoc Committee on Lupus Response Criteria. ${ }^{11}$ Patients with SLE were determined to have NPSLE with diffuse syndromes on the basis of a review of their medical records and on the data obtained from the neuropsychological, psychological, and other medical components of the study. Those patients diagnosed with a mood disorder all had major depression (mild to moderate), defined as related to the underlying NPSLE.

Neuropsychological testing was overseen by a board-certified clinical neuropsychologist. The battery of neuropsychological tests assessed cognitive function in the domains of processing speed; visual, auditory/verbal, and visual-spatial memory; sustained attention; and executive function. Processing speed and visual working memory were assessed with the oral Rao adaptation of the Symbol Digit Modalities Test. ${ }^{16,17}$ Attention and verbal working memory were assessed with the Rao adaptation of the Paced Auditory Serial Addition Task (PASAT) by using 3-second interstimulus interval conditions. ${ }^{17,18}$ Auditory/verbal memory was assessed with the California Verbal Learning Test, 2nd ed. ${ }^{19}$ Visual-spatial memory was assessed with the Brief Visuospatial Memory Test-Revised. ${ }^{20}$ Executive function was assessed with the Delis-Kaplan Executive Function System Test. ${ }^{21}$

The study was approved by an internal institutional review board, and written informed consent was obtained from all participants. All participants were compensated for their time.

\section{MR Imaging Acquisition}

All subjects were scanned on a 3T whole-body MR imaging system (Signa Excite HD; GE Healthcare, Milwaukee, Wisconsin), with a maximum slew rate of $150 \mathrm{~T} / \mathrm{m} / \mathrm{s}$ and maximum gradient amplitude in each orthogonal plane of $40 \mathrm{mT} / \mathrm{m}$ (zoom mode) by 
using an 8-channel head and neck coil. All scans were prescribed parallel to the subcallosal line in an axial-oblique orientation. The subjects were scanned with FLAIR $(\mathrm{TR}=8500 \mathrm{~ms}$, TE $=120 \mathrm{~ms}$, $\mathrm{TI}=2100 \mathrm{~ms}$, voxel size $=0.94 \times 0.94 \times 3 \mathrm{~mm}), 3 \mathrm{D}$ gradient recalled-echo $\mathrm{T} 1 \mathrm{WI}(\mathrm{TR}=24 \mathrm{~ms}, \mathrm{TE}=7 \mathrm{~ms}$, voxel size $=0.94$ $\times 0.94 \times 1.5 \mathrm{~mm})$, and DTI sequences $(\mathrm{TR}=8600 \mathrm{~ms}, \mathrm{TE}=85 \mathrm{~ms}$, $b=1000,39$ diffusion directions, voxel size $=1.1 \times 1.1 \times 3 \mathrm{~mm}$ ).

\section{MR Imaging Analysis}

Volumetric data were obtained by using 3D T1WI. Scans were adjusted by using an in-house-developed inpainting technique to avoid tissue misclassification. Structural Image Evaluation by using Normalization of Atrophy Cross-Sectional (SIENAX, Version 2.6; http:/fsl.fmrib.ox.ac.uk/fsl/fslwiki/SIENA) was used to acquire measures of normalized whole-brain and GM and WM volumes, with actual segmentation performed by the FMRIB Automated Segmentation Tool (FAST; http://fsl.fmrib.ox.ac. uk/fsl/fslwiki/fast). ${ }^{22,23}$

Lesions were evaluated on FLAIR images with reference to T2/proton-attenuation images. A semiautomated edge-detection contouring-thresholding technique was used to create subjectspecific binary lesion masks, also used in Zivadinov et al. ${ }^{24}$

The DTI was corrected for motion and eddy current distortion by using the FMRIB Diffusion Toolbox (http://www.fmrib.ox. ac.uk/fsl/fdt/index.html). ${ }^{25}$ To eliminate all nonbrain tissue, we extracted and deskulled the original $b=0$ images (with no diffusion-weighting) by using the FSL Brain Extraction Tool (http:// fsl.fmrib.ox.ac.uk/fsl/fslwiki/BET). ${ }^{26}$ On the basis of the Matlab (MathWorks, Natick, Massachusetts) code provided by the original authors, ${ }^{27}$ we applied a bi-tensor fitting to account for the partial voluming effects of free water. The FA, MD, radial diffusivity (RD), and axial diffusivity ( $A D)$ maps were calculated in Matlab by using the corrected tissue diffusion tensor as estimated by the free water elimination technique. ${ }^{28}$ Because field maps were not acquired in this study, a 2-step process was used to reduce the inherent susceptibility-induced spatial distortions in EPI-based acquisitions. First, the $b=0$ images were linearly coregistered into the same geometric space as the FLAIR images by using the FMRIB Linear Image Registration Tool (FLIRT; http://fsl.fmrib.ox.ac.uk/fsl/fslwiki/FLIRT). The images were then nonlinearly coregistered by using the Advanced Normalization Tools package (http://stnava.github.io/ANTs/). ${ }^{29}$ These 2 transformations were then applied to each of the DTI outputs so that all scalar measurements were resampled into the native FLAIR geometric space. ${ }^{14,29}$ Specific tissue compartment masks of WM (see above) were used to obtain global WM diffusion measures.

Voxelwise intergroup statistical analysis of the DTI data was performed by using TBSS. ${ }^{14}$ First, subjects' FA data were aligned into a common space by using the FMRIB Nonlinear Registration Tool (FNIRT; http://fsl.fmrib.ox.ac.uk/fsl/fslwiki/FNIRT), which uses a b-spline representation of the registration warp field. Next, the mean FA image was created and thinned to create a mean FA skeleton, which represented the centers of all tracts common to the group. Each subject's aligned FA data were then projected onto this skeleton. The same transformations and skeletonization were also applied to the other DTI metric maps, as well as to the individual subject lesion maps.

\section{Statistical Analysis}

Analyses were performed by using SPSS, Version 20.0 (IBM, Armonk, New York). Due to non-normality, the T2 lesion number and volume differences between the groups were evaluated with the Mann-Whitney $U$ test. Whole-brain and tissue-specific volume differences were evaluated by using 1-way ANOVA with post hoc Tukey correction. Parametric 1-way ANCOVAs, adjusted for age and sex, were performed to assess differences in global WM diffusion characteristics among the groups. One-way ANCOVA, adjusted for age and sex, was performed to compare the neuropsychological tests among the 3 groups, while the Bonferroni post hoc $t$ test was used to assess differences between the individual groups.

For both global WM and voxelwise TBSS analyses, the specific DTI parameters investigated were FA and MD. In the event that either was found significantly different between group comparisons $(P<.05)$, additional diffusion metrics, $\mathrm{RD}$ and $\mathrm{AD}$, were also reported. For TBSS analysis, lesions maps were also taken into consideration as voxelwise covariates, to model out their effect and focus on changes in WM in these groups. Additionally, we directly compared lesion attenuation between patients with MS and those with NPSLE along TBSS-derived tracts $(P<.05)$.

Age and sex were also used as covariates in both the TBSS and global WM analyses. For TBSS analysis, ANCOVA was performed to compare the 3 groups, with additional post hoc $t$ tests to investigate regional differences between HCs and patients with MS, HCs and patients with NPSLE, and patients with MS and NPSLE. Inference was performed by using the nonparametric FSL Randomise permutation testing tool (http://fsl.fmrib.ox.ac.uk/fsl/ fslwiki/Randomise) with 5000 permutations for each test. ${ }^{30}$ The Randomise command using the FSL General Linear Model function (http://fsl.fmrib.ox.ac.uk/fsl/fslwiki/GLM) allows the comparison of diffusion metrics among the study groups in a voxelwise fashion. The tracts were dilated by using the tbss_fill command in FSL to enhance visualization of the results. Withingroup comparisons of neuropsychological test scores with diffusion metrics (FA and MD) were performed for each subject group. Using the FSL General Linear Model function, we created matrices, including neuropsychological tests scores as voxelwise covariates, in the TBSS comparisons. The subjects' lesion data were also incorporated as voxelwise covariates in all analyses. All TBSS results were corrected for family-wise error at $P<.05$ by using threshold-free cluster enhancement to account for spatially correlated changes. ${ }^{31}$

\section{RESULTS}

\section{General Demographic and Clinical Characteristics}

Table 1 shows demographic and clinical characteristics of the 3 study groups. There were no significant differences among the study groups for age and sex. On the basis of the disease-severity scores, both subjects with NPSLE and those with MS were determined to have mild-to-moderate disease severity. The median Expanded Disability Status Scale score, used for quantifying disability in MS, was 3.3 for the patients with MS, ${ }^{32}$ while the mean Systemic Lupus Activity Measure, used for quantifying disability in SLE, was 9.9 for patients with NPSLE. ${ }^{15}$ No significant differences in disease duration were seen between the 2 patient groups. Medications taken by the study subjects are listed in Table 1 . None of the HCs were on prescribed medications. 
Of the 23 patients with NPSLE, 6 had migraine, mood disorder, and cognitive impairment; 6 had migraine and mood disorder; 5 had migraine and cognitive impairment; 2 had mood disorder and cognitive impairment; and 4 had migraine. In addition to CNS-related NPSLE syndromes, 14 of the 23 patients had peripheral involvement ( 8 had peripheral neuropathy and cranial neuropathy and 6 had peripheral neuropathy).

\section{Neuropsychological Characteristics}

Table 2 shows the neuropsychological measures of the 3 groups. Significant ANCOVA differences among the 3 groups were found for the California Verbal Learning Test, 2nd ed $(P=.001)$; the Brief Visuospatial Memory Test-Revised; $(P=.005)$, and the Delis-Kaplan Executive Function System $(P=.011)$. There was also a trend toward significance for the PASAT $(P=.061)$.

There were no significant differences in cognitive performance between the subjects with MS and those with NPSLE on any of the neuropsychological measures. Patients with MS performed significantly worse than HCs on the California Verbal Learning Test,

Table 1: Demographic and clinical characteristics of the study groups ${ }^{a}$

\begin{tabular}{lcccc}
\hline & MS $(\boldsymbol{n}=\mathbf{3 0})$ & dNPSLE $(\boldsymbol{n}=\mathbf{2 3})$ & HC $(\boldsymbol{n}=43)$ & $\boldsymbol{P}$ Value $^{\mathbf{b}}$ \\
\hline Female sex (No.) (\%) & $23(76.5 \%)$ & $22(95.7)$ & $37(86.0)$ & .150 \\
Age (yr) (mean) (SD) & $43.8(8.6)$ & $48.9(12.5)$ & $44.7(9.8)$ & .170 \\
Disease duration (yr) (mean) (SD) & $11.7(8)$ & $15(9.6)$ & NA & .237 \\
SLAM checklist (mean) (SD) & NA & $9.9(4.9)$ & NA & NA \\
EDSS (median) (IQR) & $3.3(2.1-6.0)$ & NA & NA & NA
\end{tabular}

Note:-NA indicates not applicable; dNPSLE, neuropsychiatric systemic lupus erythematosus with diffuse syndromes; SLAM, systemic lupus activity measure; EDSS, Expanded Disability Status Scale; IQR, interquartile range.

a Disease-modifying medications for treatment of MS included interferon $\beta(n=18)$. natalizumab $(n=8)$, and glatiramer acetate $(n=4)$ ). Other treatment included antianxiety (dNPSLE, $n=2 ; \mathrm{MS}, n=0$ ), antiepileptic (dNPSLE, $n=0 ; \mathrm{MS}, n=$ 5), antidepressants (dNPSLE, $n=10$ : MS, $n=11$ ), antifatigue (dNPSLE, $n=0 ; \mathrm{MS}, n=4$ ), antispastic (dNPSLE, $n=0 ; \mathrm{MS}$, $n=8$ ), antiparesthesia (dNPSLE, $n=0 ; \mathrm{MS}, n=8$ ), hydroxychloroquine (Plaquenil) (dNPSLE, $n=21$ ), imuran (dNPSLE, $n=$ 4), immunosuppressive (dNPSLE, $n=21 ; \mathrm{MS}, n=5$ ), steroids or nonsteroidal antiinflammatory drugs (dNPSLE, $n=17$; MS $n=1)$.

b The $\chi^{2}$ test was used to evaluate significant differences in sex distribution among the groups, and analysis of variance was used to evaluate significant differences in age and disease duration. 2nd ed $(P=.003)$; the Brief Visuospatial Memory Test-Revised $(P=.004)$; and the Delis-Kaplan Executive Function System $(P=$ $.010)$. There were no significant differences in performance between patients with MS and HCs on the Symbol Digit Modalities Test or the PASAT. Subjects with NPSLE performed significantly worse than HCs on the California Verbal Learning Test, 2nd ed $(P=.009)$. In general, there was a clear trend of cognitive dysfunction in patients with NPSLE compared with HCs.

\section{General Conventional MR Imaging Characteristics}

Table 3 shows lesion and brain volumetry outcomes in the 3 study roups.

Patients with MS showed a significantly higher T2 lesion volume compared with patients with NPSLE $(P<.0001$ for both). There were no significant T2 lesion number differences between patients with NPSLE and HCs. Patients with NPSLE showed significantly increased T2 lesion volume compared with $\mathrm{HCs}(P=.023)$. Figure 1 shows areas where patients with MS had significantly greater lesion attenuation than patients with NPSLE, including the left superior corona radiata and a small portion of the right posterior corona radiata.

Patients with MS showed significantly decreased whole-brain volume compared with HCs $(P<.0001)$ and also compared with patients with NPSLE $(P=.003)$. No significant whole-brain volume differences were found between patients with NPSLE and HCs.

Table 2: Comparison of subject groups on neuropsychological measures ${ }^{a}$

\begin{tabular}{lccccccc}
\hline & MS (Mean) (SD) & dNPSLE (Mean) (SD) & HC (Mean) (SD) & $\begin{array}{c}\text { ANOVA } \\
(\boldsymbol{P} \text { Value) }\end{array}$ & $\begin{array}{c}\text { MS vs HC } \\
(\boldsymbol{P} \text { Value) }\end{array}$ & $\begin{array}{c}\text { dNPSLE vs HC } \\
(\boldsymbol{P} \text { Value) }\end{array}$ & $\begin{array}{c}\text { MS vs dNPSLE } \\
(\boldsymbol{P} \text { Value) }\end{array}$ \\
\hline SDMT & $54.6(16.1)$ & $54.8(10.3)$ & $60.7(10.4)$ & .091 & .137 & .373 & 1.0 \\
CVLT2 & $49.3(12.3)$ & $50.35(9.7)$ & $57.9(7.9)$ & .001 & .003 & .009 & 1.0 \\
BVMT-R & $21.7(7.4)$ & $24.3(6.9)$ & $26.9(5.7)$ & .005 & .004 & .571 & .359 \\
D-KEFS & $9.2(2.8)$ & $10.3(2.6)$ & $119(2.2)$ & .011 & .010 & .252 & .722 \\
PASAT & $41.6(16)$ & $42(12.3)$ & $48.3(12.3)$ & .061 & .111 & .226 & 1.0 \\
\hline
\end{tabular}

Note:-CVLT2 indicates California Verbal Learning Test, 2nd ed; BVMT-R, Brief Visuospatial Memory Test-Revised; D-KEFS, Delis-Kaplan Executive Function System; SDMT, Symbol Digit Modalities Test; dNPSLE, neuropsychiatric systemic lupus erythematosus with diffuse syndromes.

a Reported measures are raw scores. The statistical analysis among the groups was performed using l-way analysis of covariance, adjusted for age and sex. Post hoc tests between the individual groups were performed using a Bonferroni correction.

Table 3: Comparison of conventional MRI characteristics among the study groups ${ }^{\mathrm{a}}$

\begin{tabular}{lcccccc}
\hline & MS ( $\boldsymbol{n}=\mathbf{3 0})$ & dNPSLE ( $\boldsymbol{n = 2 3 )}$ & HC ( $\boldsymbol{n = 4 3 )}$ & $\begin{array}{c}\text { MS vs HCs }^{\text {b }} \\
(\boldsymbol{P} \text { Value) }\end{array}$ & $\begin{array}{c}\text { dNPSLE vs HCS }^{\mathbf{c}} \\
(\boldsymbol{P} \text { Value) }\end{array}$ & $\begin{array}{c}\text { MS vs dNPSLE }^{\mathbf{d}} \\
(\boldsymbol{P} \text { Value) }\end{array}$ \\
\hline T2 lesion number (mean) (SD) & $31.1(19.2)$ & $11.7(9.3)$ & $7.9(4.8)$ & $<.0001$ & .06 & $<.0001$ \\
T2 lesion volume (mean) (SD) & $15.2(3.1)$ & $1.9(1.3)$ & $1.6(.4)$ & $<.0001$ & .023 & $<.0001$ \\
Normalized WB volume (mean) (SD) & $1490.4(113.5)$ & $1570.3(79)$ & $1596.5(64.2)$ & $<.0001$ & .465 & .003 \\
Normalized WM volume (mean) (SD) & $784.5(58.0)$ & $829.3(45.1)$ & $849.0(40.8)$ & $<.0001$ & .285 & .005 \\
Normalized GM volume (mean) (SD) & $704.0(63.9)$ & $751.5(58.8)$ & $755.3(42)$ & .001 & .964 & .008 \\
\hline
\end{tabular}

Note:-WB indicates whole-brain; dNPSLE, neuropsychiatric systemic lupus erythematosus with diffuse syndromes.

a The statistical analysis among the groups was performed using 1-way analysis of variance with post hoc Tukey correction, except for the $\mathrm{T} 2$ lesion number and volume, which were analyzed using the Mann-Whitney $U$ test. All volumes are given in milliliters.

${ }^{\mathrm{b}} \mathrm{P}$ value for MS vs $\mathrm{HCs}$.

c $P$ value for SLE vs HCs.

${ }^{d} P$ value for MS vs SLE. 


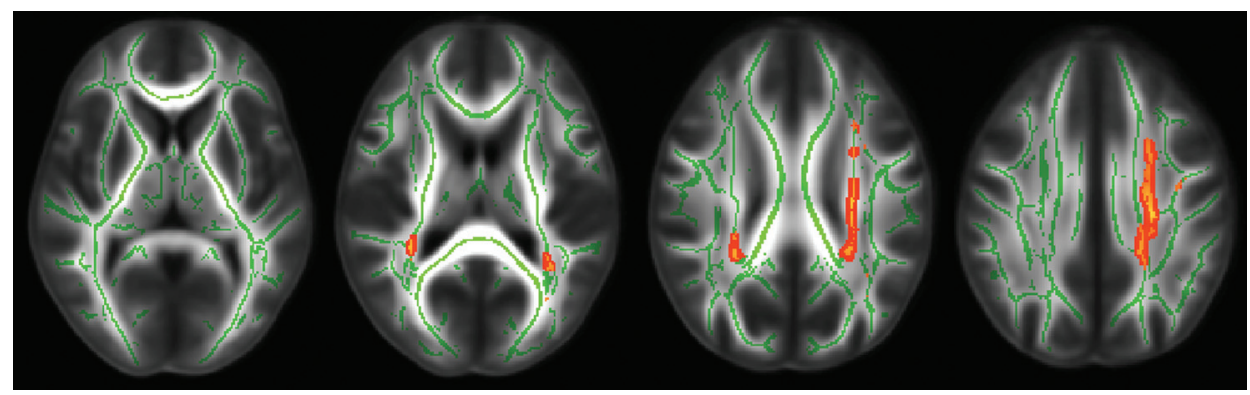

FIG 1. A comparison of lesion attenuation along WM tracts in patients with MS and those with NPSLE by using TBSS analysis. Red-highlighted areas show where patients with MS had significantly greater lesion attenuation than patients with NPSLE $(P<.05)$. Patients with MS have significant lesion attenuation as shown in the left superior corona radiata and a small portion of the right posterior corona radiata.

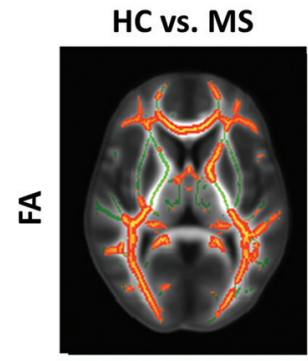

HC vs. dNPSLE

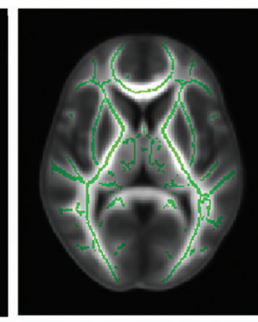

dNPSLE vS. MS
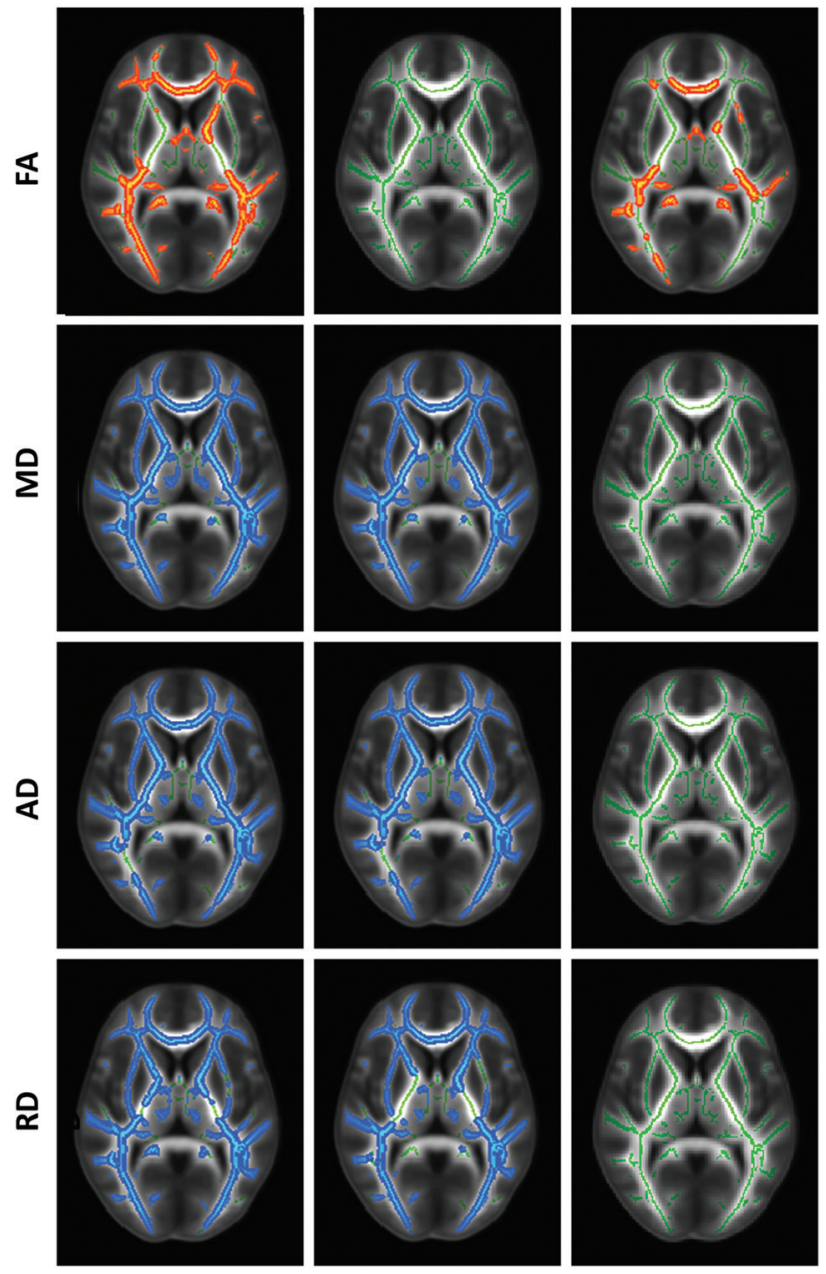

FIG 2. Diffusion tensor imaging TBSS differences among patients with MS, patients with NPSLE with diffuse syndromes, and HCs, controlling for age and sex. The results are shown for FA, MD, RD, and AD differences between the studied groups $(P<.05)$. Red-highlighted areas show where significantly lower FA was found in the second reported group, indicating less anisotropic diffusion. Blue-highlighted areas show significantly increased diffusivity in the second reported group, indicating more diffuse WM damage.

Patients with MS showed significantly decreased WM volume compared with HCs $(P<.0001)$. The patients with MS also showed significantly decreased WM volume compared with patients with NPSLE $(P=.005)$. No significant WM volume differences were found between patients with NPSLE and HCs.
Patients with MS showed significantly decreased GM volume compared with patients with NPSLE $(P=.008)$ and also compared with HCs $(P=.001)$. No significant GM volume differences were found when comparing patients with NPSLE and HCs.

\section{Diffusion Tensor Imaging White Matter Characteristics among the Study Groups}

Figure 2 summarizes the results of WM tract differences by using the TBSS analysis. Highlighted areas represent significant diffusivity differences among the 3 study groups corrected for multiple comparisons at the family-wise $P<.05$ level on the threshold-free cluster-enhanced images. Table 4 shows the global WM differences among the study groups.

In the TBSS analysis comparing patients with MS and HCs, patients with MS showed significant widespread damage throughout WM tracts across all diffusion metrics without any specific regional predilection for $\mathrm{FA}, \mathrm{MD}, \mathrm{AD}$, and $\mathrm{RD}$ (Fig 2, left column). Global WM measures also revealed significantly different diffusivity characteristics across all diffusivity metrics (FA, $P=.001 ; \mathrm{MD}, P<.0001$; $\mathrm{AD}, P=.006$; and $\mathrm{RD}, P<.0001$; Table 4 ).

TBSS contrasts between patients with NPSLE and HCs also revealed widespread differences in WM tract diffusivity in MD, $\mathrm{RD}$, and $\mathrm{AD}$ measures; however, no significant differences were found for FA (Fig 2, middle column). Global WM measures also revealed significant differences for $\mathrm{MD}(P=.001), \mathrm{AD}(P=.003)$, and $\mathrm{RD}(P<.0001)$, but not for FA (Table 4$)$.

TBSS contrasts between patients with MS and those with NPSLE showed prevalent FA differences throughout central WM pathways, including the corpus callosum; the inferior longitudinal and fronto-occipital fasciculi; and also parts of the forceps major, forceps minor, cingulum, and thalamic radiation (Fig 2, right column). Figure 3 provides additional sagittal and coronal views of decreased FA in patients with MS compared with patients with NPSLE. There were no significant differences in TBSS analysis between patients with MS and those with NPSLE for MD, AD, and RD measures. Global WM measures showed no significant differences between patients with MS and those with NPSLE for any diffusion metrics.

\section{White Matter Tract Integrity and Cognitive Function Associations among the Study Groups}

Figure 4 shows the results of within-group comparisons of cognitive performance and WM tract integrity diffusion metrics for each subject group. 
Table 4: Comparison of global white matter diffusion tensor imaging characteristics among the study groups ${ }^{\mathrm{a}}$

\begin{tabular}{|c|c|c|c|c|c|c|}
\hline & MS & dNPSLE & $\mathrm{HC}$ & MS vs $\mathrm{HCs}^{\mathrm{b}}$ & dNPSLE vs $\mathrm{HCs}^{\mathrm{c}}$ & MS vs dNPSLE ${ }^{d}$ \\
\hline FA (mean) (SD) & $.398(.006)$ & $.411(.004)$ & $.418(.002)$ & .001 & .112 & .310 \\
\hline $\mathrm{MD} \times 10^{-3}$ (mean) $(\mathrm{SD})$ & $.786(.009)$ & $.775(.008)$ & $.734(.008)$ & $<.0001$ & .001 & .780 \\
\hline $\mathrm{AD} \times 10^{-3}$ (mean) $(\mathrm{SD})$ & $1.149(.010)$ & $1.150(.012)^{\prime}$ & $1.096(.013)$ & .006 & .003 & .784 \\
\hline $\mathrm{RD} \times 10^{-3}$ (mean) $(\mathrm{SD})$ & $.604(.01)$ & $.587(.006)$ & $.553(.006)$ & $<.0001$ & $<.0001$ & .528 \\
\hline
\end{tabular}

Note:- dNPSLE indicates neuropsychiatric systemic lupus erythematosus with diffuse syndromes.

${ }^{a}$ The statistical analysis between the groups was performed using 1-way analysis of variance controlling for age and sex. FA is a dimensionless measure. Diffusivity is given in square millimeters second ${ }^{-1}$.

${ }^{b} P$ value for MS vs HCs.

c $P$ value for SLE vs HCS.

${ }^{d} P$ value for MS vs SLE

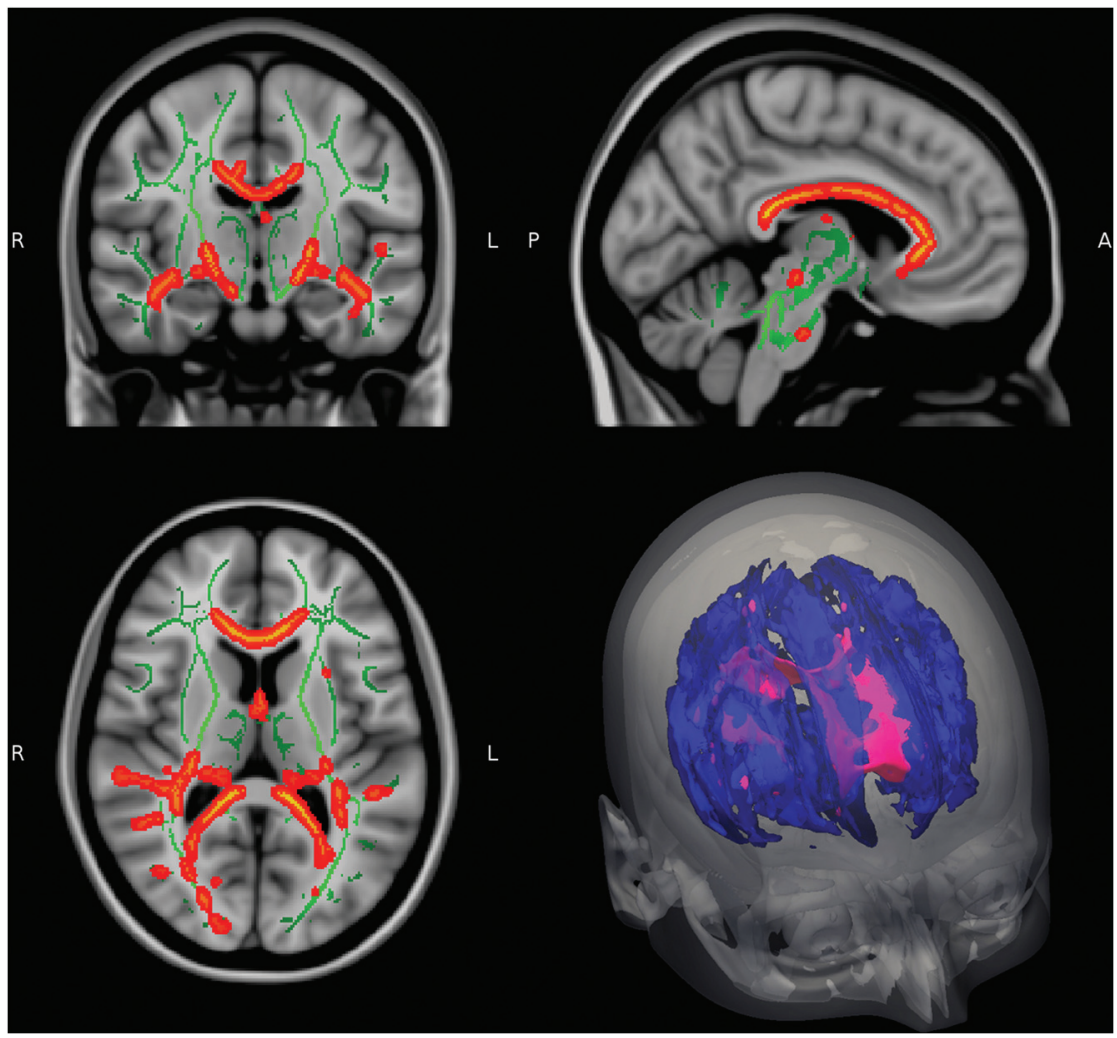

FIG 3. Details of TBSS FA comparison between patients with MS and those with NPSLE with diffuse syndromes. Red areas show where FA is significantly lower in patients with MS compared with patients with NPSLE in central white matter pathways, including the corpus callosum, inferior longitudinal fasciculus, and fronto-occipital fasciculus $(P<.05)$.

Lower cognitive scores in patients with MS were significantly associated with decreased FA and MD for all examined domains, while in patients with NPSLE, only decreased FA in the superior WM pathways was significantly associated with executive functioning (Delis-Kaplan Executive Function System). Lower PASAT scores were significantly associated with increased MD in the HC group.

\section{DISCUSSION}

While the full extent of NPSLE and MS pathology is not completely understood, both diseases involve inflammatory autoimmune processes that lead to axonal loss with consequent neurologic impairment and deficits in cognitive performance. ${ }^{33,34}$ Numerous studies have also revealed WM abnormalities in patients with NPSLE, ${ }^{35,36}$ including the presence of WM lesions ${ }^{37}$ and increased WM tract diffusivity. ${ }^{38}$ The pathogenesis of CNS involvement in SLE is likely related to an inflammatory response secondary to auto-antibody-mediated vasculitis. ${ }^{35}$

The increased sensitivity in DTI has proved useful for detecting WM tract deterioration. ${ }^{39-41}$ DTI allows quantitative measurements of diffusion anisotropy along WM tracts. FA is a widely used measure that reflects the degree of directionality of water diffusion. For example, the myelin within intact WM tracts generally is uniformly oriented parallel to the overall tracts, and molecular water movement is much greater parallel to the tracts than perpendicular to the tracts. This myelin orientation results in highly directional, anisotropic diffusion and therefore high FA values. However, as tracts become damaged due to demyelination or other pathologic processes, the tissue structure becomes less orderly and FA decreases as water diffuses more freely in multiple directions. While FA reflects the directionality of diffusion, $\mathrm{MD}$ reflects the magnitude of diffusion. $\mathrm{AD}$ corresponds to the diffusivity along the principal axis, whereas RD corresponds to the average of the diffusivities along the 2 minor axes. ${ }^{14,42}$

The results of this study are consistent with those in prior studies showing significant WM damage in patients with MS compared with HCs across measures of FA, MD, RD, and AD, by using both TBSS and global WM analysis. ${ }^{33,43}$ The results are also consistent with prior studies showing that subjects with MS perform significantly worse than HCs on cognitive tests assessing processing speed, working and visual-spatial processing/memory, sustained attention, and executive function. ${ }^{21,44-46}$

The diffuse SLE syndromes such as anxiety disorders, mood disorders, headache, and cognitive impairment may be the result of diffuse damage, whereas focal SLE syndromes such as seizures, strokes, and transient ischemic attacks, result in high-attenuation focal lesions. Several studies have shown regionally-specific decreased FA and increased MD in patients with NPSLE compared with HCs. ${ }^{38,47}$ In our comparison of patients with diffuse NPSLE and $\mathrm{HCs}$, we found significant differences in $\mathrm{MD}, \mathrm{AD}$, and $\mathrm{RD}$ in both the TBSS and global WM measures; however, we did not 
MS

FA
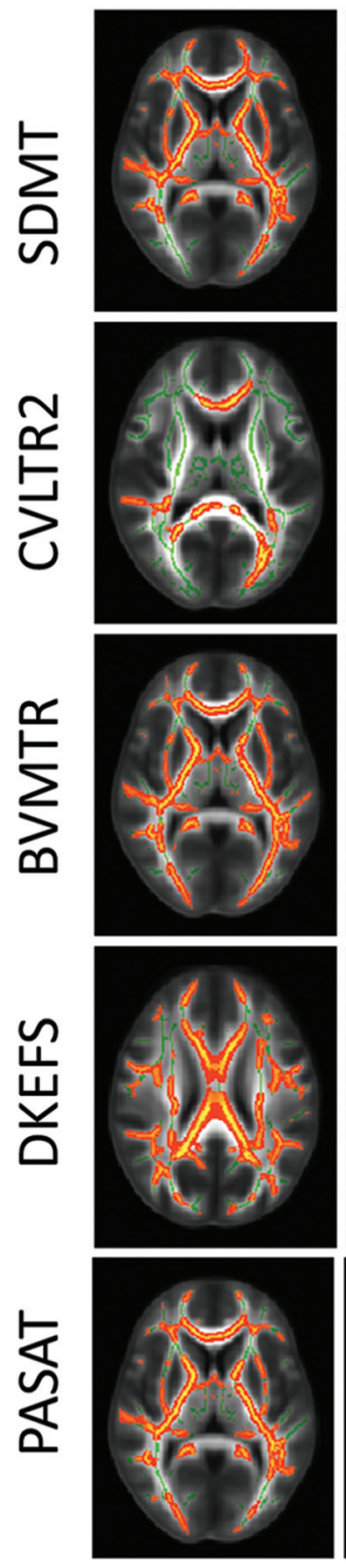

dNPSLE
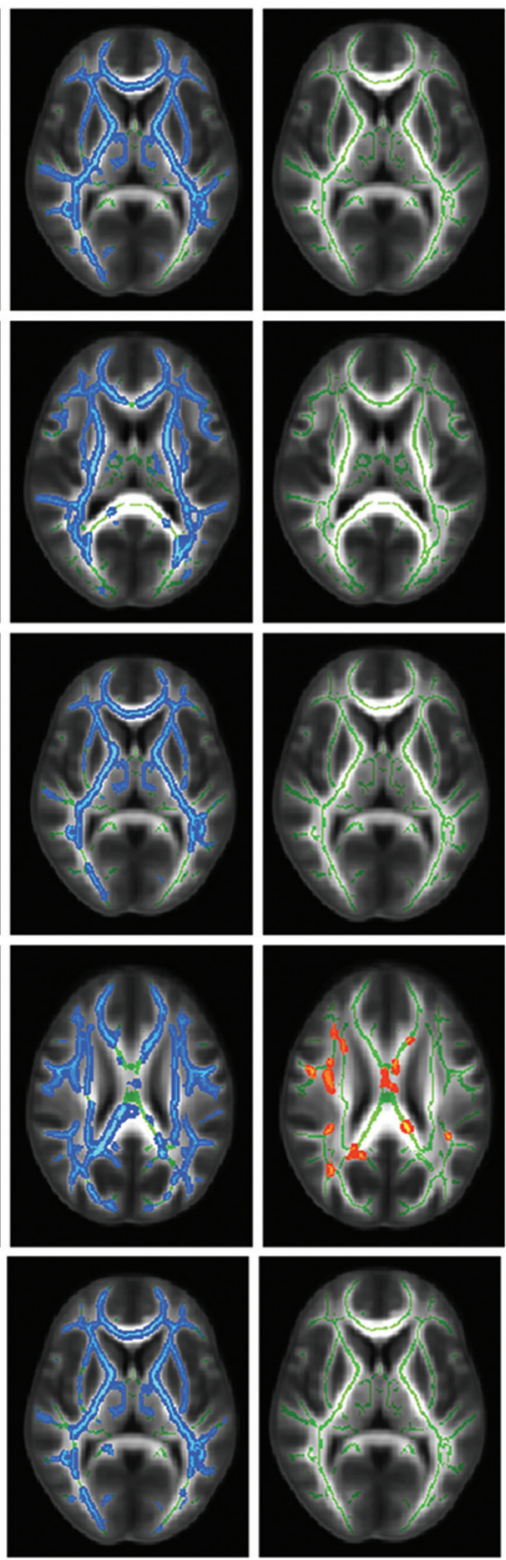

FA

MD
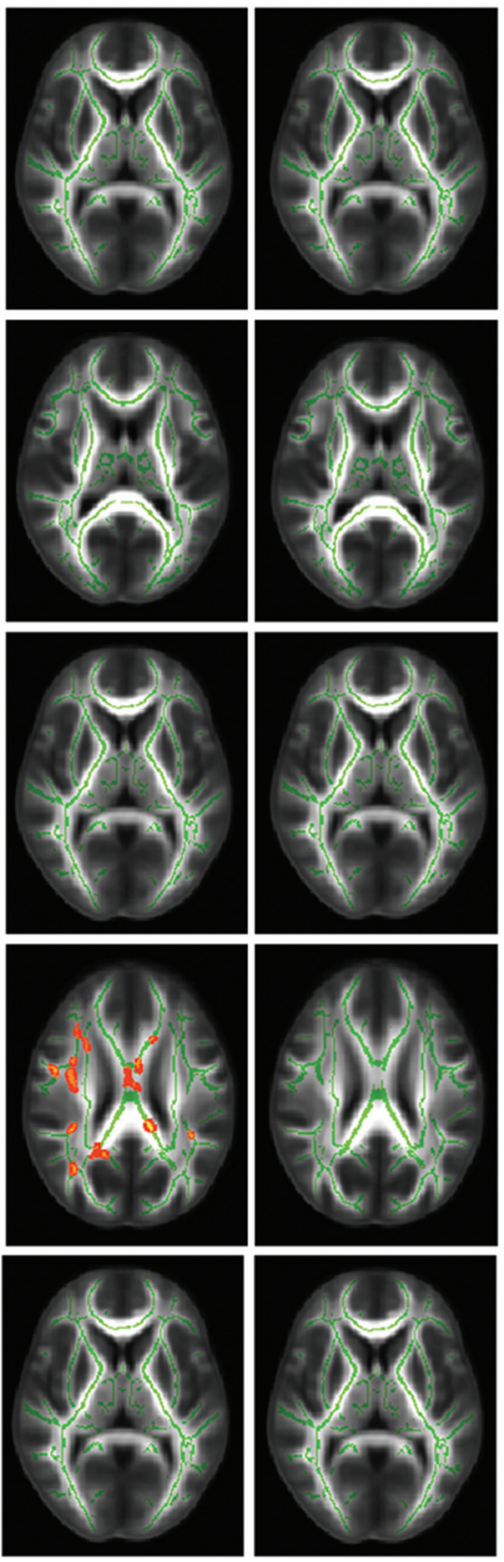
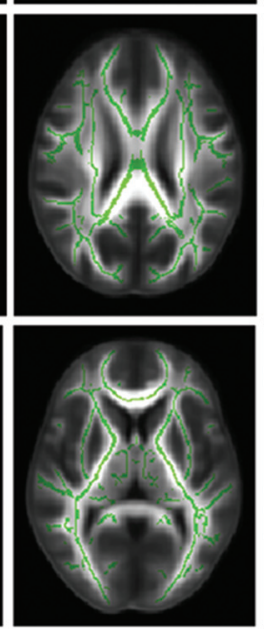

HC

FA
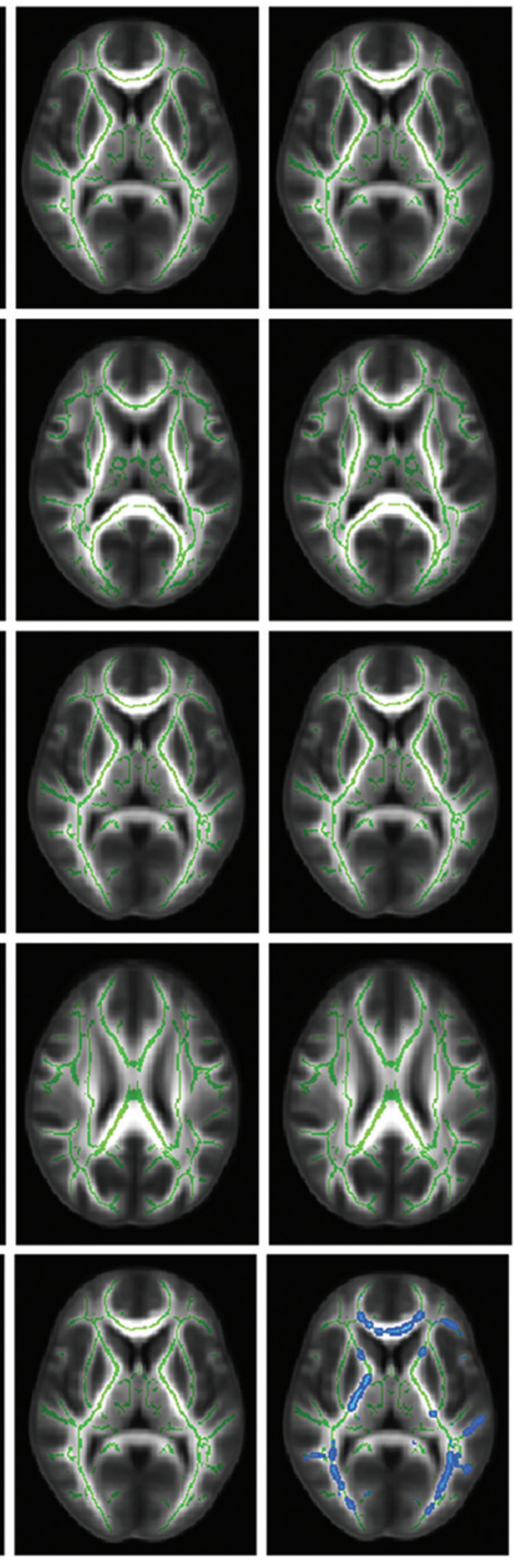

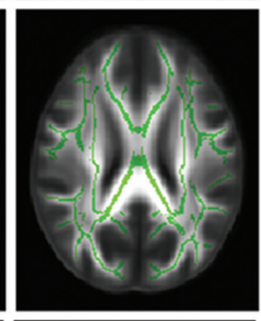

MD
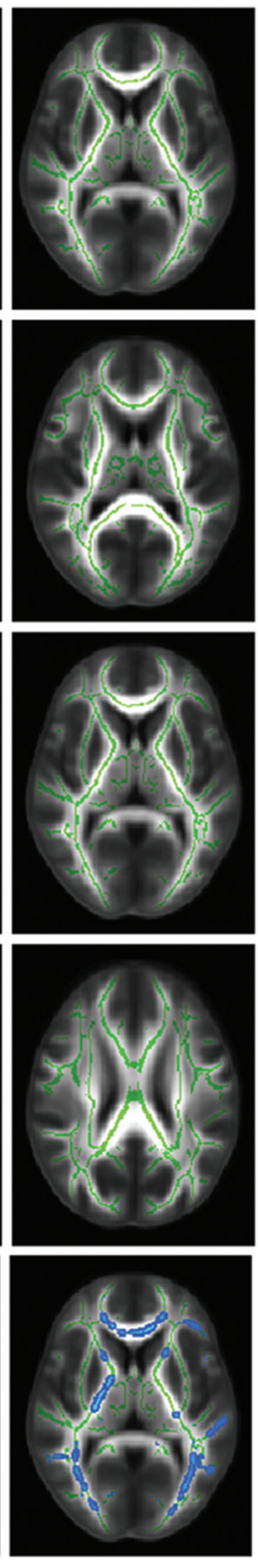

FIG 4. Highlighted tracts display significant correlations between TBSS differences and performance on neuropsychological tests $(P<.05)$. Red-highlighted tracts represent positive correlations, and blue-highlighted tracts represent negative correlations. For patients with MS, decreased neuropsychological scores significantly correlated with decreased FA and increased MD for each of the neuropsychological tests. For patients with NPSLE, decreased Delis-Kaplan Executive Function System scores significantly correlated with decreased FA. For HCs, increased PASAT scores correlated significantly with decreased MD. CVLT2 indicates California Verbal Learning Test, 2nd ed; BVMT-R, Brief Visuospatial Memory Test-Revised; D-KEFS, Delis-Kaplan Executive Function System; SDMT, Symbol Digit Modalities Test; dNPSLE, neuropsychiatric systemic lupus erythematosus with diffuse syndromes.

detect differences in FA in either of the analyses. These findings may be because the present study included only subjects with SLE with diffuse neuropsychiatric syndromes, unlike the previous studies, which included patients with focal neuropsychiatric SLE syndromes. Diffusivity changes in patients with NPSLE might be due to increased extracellular water, resulting in more isotropic WM changes affecting both RD and AD; this increased extracellular water would account for differences in MD, but not in FA, between patients with NPSLE and HCs.
No significant differences were found between patients with NPSLE and HCs in WM volume. This finding is consistent with a recent study by Zivadinov et $\mathrm{al}^{10}$ but contrary to the study of Appenzeller et al. ${ }^{12}$ In addition, no differences were found between patients with NPSLE and HCs in GM volume, contrary to findings in our previously published study. ${ }^{10}$

Prior studies of cognitive impairment in SLE have most commonly found deficits in processing speed and working and verbal memory. ${ }^{3,8,48}$ Kozora et al ${ }^{49}$ also found that subjects with SLE 
showed impaired working memory on the PASAT, which inversely correlated to WM abnormalities. In this study, patients with NPSLE generally performed worse than HCs on all the administered cognitive tests, though the differences were only significant for auditory and verbal memory.

In the TBSS analysis comparing patients with MS with those with NPSLE, there was more diffuse damage in MS as evidenced by decreased FA throughout the central WM, including the corpus callosum and the inferior longitudinal and fronto-occipital fasciculi. However, no significant differences between patients with NPSLE and patients with MS were found for MD, AD, or RD; and the global WM measures failed to reveal differences in these diffusivity metrics.

It is possible that the positive findings of FA differences between MS and NPSLE and negative findings of MD, AD, and RD merely reflect insufficient statistical power. However, given the reasonably large group sizes involved, one should consider the true biologic mechanisms for this seemingly contradictory result. Increased RD has been shown to be a marker of decreased myelin content in postmortem MS brains. ${ }^{50}$ Studies using MS mouse models have also established that changes in $\mathrm{RD}$ are attributed to myelin damage and additionally that $\mathrm{AD}$ characteristics are a strong predictor of axonal attenuation. ${ }^{51,52}$ Recent studies suggest that while axonal loss is characteristic of NPSLE pathology, demyelination is a less specific finding in patients with NPSLE. ${ }^{34,53}$ Although not significant between the disease groups, in MS versus $\mathrm{HC}$, RD was increased by $9.2 \%$ compared with $4.8 \%$ for AD. In contrast, in NPSLE, RD was only increased by $6.1 \%$, closer to the 4.9\% difference in $\mathrm{AD}$.

In patients with MS, it is likely that cognitive deficits are associated with the loss of WM integrity. However, in patients with NPSLE, the explanation for cognitive deficits was less clear. Executive function was the only domain that showed a significant association with decreased WM integrity. However, the lack of association between diffusivity and performance of other cognitive domains may be related to greater local anatomic heterogeneity of WM damage in the subjects. Most surprising, HCs also showed a correlation between MD and cognitive performance on the PASAT. It would be interesting in the near future to explore changes of diffusivity and attention and processing speed in relation to the age and sex of healthy individuals.

There are some limitations in the current study. Ideally, diffusion images can be corrected by field maps, which analytically remove distortion errors, or by the acquisition of 2 datasets with opposite phase-encoding directions, which can correct for both distortion errors and intensity artifacts. We were not able to acquire data in this way during clinical routine scanning and so used an ad hoc technique of nonlinearly aligning to FLAIR. While this corrects substantially for spatial distortions, it cannot recover lost intensity information near sinuses and other air/bone interfaces. Another limitation is that both MS and NPSLE had different clinical disease characteristics, so matching these 2 types of patients from the disease severity point of view is a difficult task. However, we matched the patients for disease duration and disease severity on the basis of the respective criteria for each condition, which certainly does not overcome the elimination of differences between the 2 disease states.

\section{CONCLUSIONS}

MS has provided an interesting comparison for the less studied disease, NPSLE. Our analyses confirmed the results of prior studies showing that patients with both MS and NPSLE experience significant decreases in WM tract integrity, compared with HCs. Global WM measures comparing patients with MS and those with NPSLE showed no significant differences, but more specific TBSS investigation indicated FA differences specifically in the central WM pathways between the groups. The decrease in WM tract integrity was associated with reduced cognitive function in all explored cognitive domains only in patients with MS, while the decline in verbal and auditory memory in patients with NPSLE was not associated with altered WM integrity. A larger and longitudinal study would be necessary to better evaluate how changes in MR imaging findings correlate with cognitive dysfunction in patients with NPSLE.

\section{ACKNOWLEDGMENTS}

We thank Ofer Pasternak for allowing us to use his free water elimination code.

Disclosures: Michael G. Dwyer-UNRELATED: Consultancy: EMD Serono, Claret Medical, Comments: Michael Dwyer has served as a consultant for Claret Medical and on a scientific advisory board for EMD Serono; Grants/Grants Pending: Novartis, Comments: Michael Dwyer has received research grant support from Novartis. Janet L. Shucard — RELATED: Grant: National Institutes of Health*; Support for Travel to Meetings for the Study or Other Purposes: National Institutes of Health.* Ralph H.B. Benedict-UNRELATED: Consultancy: Biogen, Genentech, Genzyme, Sanofi, Adamas; Grants/Grants Pending: Biogen, Novartis, Genzyme, Acorda, Questcor; Payment for Lectures (including service on Speakers Bureaus): EMD Serono; Royalties: Psychological Assessment Resources; Other: Ralph H.B. Benedict received research support from Biogen Idec and Shire and serves on advisory panels for Bayer, Biogen, Novartis, and Actelion. Bianca Weinstock-Guttman-UNRELATED: Consultancy: Biogen Idec, Teva Neuroscience, EMD Serono, Novartis, Genzyme, Sanof; Grants/Grants Pending: Biogen Idec, ${ }^{*}$ Teva Neuroscience, ${ }^{*}$ EMD Serono, ${ }^{*}$ Novartis, * Genzyme, Sanofi*; Payment for Lectures (including service on Speakers Bureaus): Biogen Idec, Teva Neuroscience, EMD Serono, Novartis, Genzyme, Sanofi; Other: Bianca Weinstock-Guttman received honoraria as a speaker and as a consultant for Biogen Idec, Teva Pharmaceuticals, EMD Serono, Pfizer, Novartis, and Acorda. Dr Weinstock-Guttman received research funds from Biogen Idec, Teva Pharmaceuticals, EMD Serono, Pfizer, Novartis, Acorda, and Cyberonics. David W. ShucardRELATED: Grant: National Institutes of Health-National Institute of Neurological Disorders and Stroke, ${ }^{*}$ Comments: grant title: Neurological Indices of CNS Involvement in "Non CNS" SLE; Support for Travel to Meetings for the Study or Other Purposes: National Institutes of Health-National Institute of Neurological Disorders and Stroke. * Robert Zivadinov—UNRELATED: Consultancy: Teva, Genzyme, Biogen, Novartis, Claret Medical; Grants/Grants Pending: Teva, Genzyme, Biogen, Novartis, Claret Medical; Payment for Lectures (including service on Speakers Bureaus): Teva, Genzyme, Biogen, Novartis, Claret Medical. *Money paid to the institution.

\section{REFERENCES}

1. Rovaris M, Viti B, Ciboddo G, et al. Cervical cord magnetic resonance imaging findings in systemic immune-mediated diseases. J Neurol Sci 2000;176:128-30

2. Kozora E, Hanly JG, Lapteva L, et al. Cognitive dysfunction in systemic lupus erythematosus: past, present, and future. Arthritis Rheum 2008;58:3286-98

3. Benedict RH, Shucard JL, Zivadinov R, et al. Neuropsychological impairment in systemic lupus erythematosus: a comparison with multiple sclerosis. Neuropsychol Rev 2008;18:149-66

4. Fulford KW, Catterall RD, Delhanty JJ, et al. A collagen disorder of the nervous system presenting as multiple sclerosis. Brain 1972;95:373-86

5. Parmenter BA, Shucard JL, Shucard DW. Information processing deficits in multiple sclerosis: a matter of complexity. J Int Neuropsychol Soc 2007;13:417-23 
6. Shucard JL, Lee WH, Safford AS, et al. The relationship between processing speed and working memory demand in systemic lupus erythematosus: evidence from a visual n-back task. Neuropsychology 2011;25:45-52

7. Kozora E, West SG, Kotzin BL, et al. Magnetic resonance imaging abnormalities and cognitive deficits in systemic lupus erythematosus patients without overt central nervous system disease. Arthritis Rheum 1998;41:41-47

8. Covey TJ, Shucard JL, Shucard DW, et al. Comparison of neuropsychological impairment and vocational outcomes in systemic lupus erythematosus and multiple sclerosis patients. J Int Neuropsychol Soc 2012;18:530-40

9. Kozora E, Arciniegas DB, Filley CM, et al. Cognition, MRS neurometabolites, and MRI volumetrics in non-neuropsychiatric systemic lupus erythematosus: preliminary data. Cogn Behav Neurol 2005;18:159-62

10. Zivadinov R, Shucard J, Hussein S, et al. Multimodal imaging in systemic lupus erythematosus patients with diffuse neuropsychiatric involvement. Lupus 2013;22:675-83

11. The American College of Rheumatology nomenclature and case definitions for neuropsychiatric lupus syndromes. Arthritis Rheum 1999;42:599-608

12. Appenzeller S, Bonilha L, Rio PA, et al. Longitudinal analysis of gray and white matter loss in patients with systemic lupus erythematosus. Neuroimage 2007;34:694-701

13. Jung RE, Chavez RS, Flores RA, et al. White matter correlates of neuropsychological dysfunction in systemic lupus erythematosus. PLoS One 2012;7:e28373

14. Smith SM, Jenkinson M, Johansen-Berg H, et al. Tract-based spatial statistics: voxelwise analysis of multi-subject diffusion data. Neuroimage 2006;31:1487-505

15. Liang MH, Socher SA, Larson MG, et al. Reliability and validity of six systems for the clinical assessment of disease activity in systemic lupus erythematosus. Arthritis Rheum 1989;32:1107-18

16. Smith A. Symbol Digit Modalities Test: Manual. Torrance: Western Psychological Services; 1982

17. Rao SM. A Manual for the Brief, Repeatable Battery of Neuropsychological Tests in Mulitple Sclerosis. Washington, DC: National Multiple Sclerosis Society; 1991

18. Gronwall DM. Paced auditory serial addition task: a measure of recovery from concussion. Percept Mot Skills 1977;44:367-73

19. Delis D, Kramer JH, Kaplan E, et al. California Verbal Learning Test Manual, 2nd ed. San Antonio, TX: Psychological Corporation; 2000

20. Benedict RH. Brief Visuospatial Memory Test-Revised: Professional Manual. Odessa, FL: Psychological Assessment Resources; 1997

21. Roca M, Torralva T, Meli F, et al. Cognitive deficits in multiple sclerosis correlate with changes in fronto-subcortical tracts. Mult Scler 2008;14:364-69

22. Zhang Y, Brady M, Smith S. Segmentation of brain MR images through a hidden Markov random field model and the expectation-maximization algorithm. IEEE Trans Med Imaging 2001;20:45-57

23. Smith SM, Jenkinson M, Woolrich MW, et al. Advances in functional and structural MR image analysis and implementation as FSL. Neuroimage 2004;23(suppl 1):S208-19

24. Zivadinov R, Heininen-Brown M, Schirda CV, et al. Abnormal subcortical deep-gray matter susceptibility-weighted imaging filtered phase measurements in patients with multiple sclerosis: a case-control study. Neuroimage 2012;59:331-39

25. Behrens TE, Johansen-Berg H, Woolrich MW, et al. Non-invasive mapping of connections between human thalamus and cortex using diffusion imaging. Nat Neurosci 2003;6:750-57

26. Smith SM. Fast robust automated brain extraction. Hum Brain Mapp 2002;17:143-55

27. Basser PJ, Pierpaoli C. Microstructural and physiological features of tissues elucidated by quantitative-diffusion-tensor MRI. J Magn Reson B 1996;111:209-19
28. Pasternak O, Sochen N, Gur Y, et al. Free water elimination and mapping from diffusion MRI. Magn Reson Med 2009;62:717-30

29. Avants BB, Tustison NJ, Song G, et al. A reproducible evaluation of ANTs similarity metric performance in brain image registration. Neuroimage 2011;54:2033-44

30. Winkler AM, Ridgway GR, Webster MA, et al. Permutation inference for the general linear model. Neuroimage 2014;92:381-97

31. Smith SM, Nichols TE. Threshold-free cluster enhancement: addressing problems of smoothing, threshold dependence and localisation in cluster inference. Neuroimage 2009;44:83-98

32. Kurtzke JF. Rating neurologic impairment in multiple sclerosis: An expanded disability status scale (EDSS). Neurology 1983;33:1444-52

33. Rovaris M, Fillippi M. Diffusion tensor MRI in multiple sclerosis. J Neuroimaging 2007:17(suppl 1)27S-30S

34. Magro Checa C, Cohen D, Bollen EL, et al. Demyelinating disease in SLE: is it multiple sclerosis or lupus? Best Pract Res Clin Rheumatol 2013;27:405-24

35. Ramage AE, Fox PT, Brey RL, et al. Neuroimaging evidence of white matter inflammation in newly diagnosed systemic lupus erythematosus. Arthritis Rheum 2011;63:3048-57

36. Bosma GP, Rood MJ, Zwinderman AH, et al. Evidence of central nervous system damage in patients with neuropsychiatric systemic lupus erythematosus, demonstrated by magnetization transfer imaging. Arthritis Rheum 2000;43:48-54

37. Appenzeller S, Vasconcelos Faria A, Li LM, et al. Quantitative magnetic resonance imaging analyses and clinical significance of hyperintense white matter lesions in systemic lupus erythematosus patients. Ann Neurol 2008;64:635-43

38. Hughes M, Sundgren PC, Fan X, et al. Diffusion tensor imaging in patients with acute onset of neuropsychiatric systemic lupus erythematosus: a prospective study of apparent diffusion coefficient, fractional anisotropy values, and eigenvalues in different regions of the brain. Acta Radiol 2007;48:213-22

39. Zivadinov R, Cox JL. Neuroimaging in multiple sclerosis. Int Rev Neurobiol 2007;79:449-74

40. Abda EA, Selim ZI, Radwan ME, et al. Markers of acute neuropsychiatric systemic lupus erythematosus: a multidisciplinary evaluation. Rheumatol Int 2013;33:1243-53

41. Cercignani M, Iannucci G, Rocca MA, et al. Pathologic damage in MS assessed by diffusion-weighted and magnetization transfer MRI. Neurology 2000;54:1139-44

42. Beaulieu C. The basis of anisotropic water diffusion in the nervous system: a technical review. NMR Biomed 2002;15:435-55

43. Yu HJ, Christodoulou C, Bhise V, et al. Multiple white matter tract abnormalities underlie cognitive impairment in RRMS. Neuroimage 2012;59:3713-22

44. Denney DR, Sworowski LA, Lynch SG. Cognitive impairment in three subtypes of multiple sclerosis. Arch Clin Neuropsychol 2005;20:967-81

45. Covey TJ, Zivadinov R, Shucard JL, et al. Information processing speed, neural efficiency, and working memory performance in multiple sclerosis: differential relationships with structural magnetic resonance imaging. J Clin Exp Neuropsychol 2011;33:1129-45

46. Vleugels L, Lafosse C, van Nunen A, et al. Visuoperceptual impairment in multiple sclerosis patients diagnosed with neuropsychological tasks. Mult Scler 2000;6:241-54

47. Emmer BJ, Veer IM, Steup-Beekman GM, et al. Tract-based spatial statistics on diffusion tensor imaging in systemic lupus erythematosus reveals localized involvement of white matter tracts. Arthritis Rheum 2010;62:3716-21

48. Kozora E, Filley CM. Cognitive dysfunction and white matter abnormalities in systemic lupus erythematosus. J Int Neuropsychol Soc 2011;17:385-92

49. Kozora E, Arciniegas DB, Duggan E, et al. White matter abnormalities and working memory impairment in systemic lupus erythematosus. Cogn Behav Neurol 2013;26:63-72

50. Schmierer K, Wheeler-Kingshott CA, Tozer DJ, et al. Quantitative 
magnetic resonance of postmortem multiple sclerosis brain before and after fixation. Magn Reson Med 2008;59:268-77

51. Song SK, Yoshino J, Le TQ, et al. Demyelination increases radial diffusivity in corpus callosum of mouse brain. Neuroimage 2005;26:132-40

52. Budde MD, Kim JH, Liang HF, et al. Axonal injury detected by in vivo diffusion tensor imaging correlates with neurological disability in a mouse model of multiple sclerosis. NMR Biomed 2008;21:589-97

53. Bertsias GK, Boumpas DT. Pathogenesis, diagnosis and management of neuropsychiatric SLE manifestations. Nat Rev Rheumatol 2010;6:358-67 